\author{
Annual Report \\ Feasibility and Demonstration of a Cloud-Based RIID Analysis System \\ Project numbers: \\ SL13-FY13-187-PD03 \\ OR13-CldBasedRIIDS-PD03
}

Lab: Oak Ridge National Laboratory

Project PI: Michael Wright

Primary Author: Michael Wright

Contributors: Michael Wright, Eric Sword, Jim Younkin

Date: 20130930

Lab: Sandia National Laboratories

Project PI: William Johnson

Primary Author: Kristen Hertz

Contributors: Kristen Hertz, Will Johnson, Lorraine Sadler, Lee Harding

Date: 20130930 


\section{Feasibility and Demonstration of a Cloud-Based RIID Analysis System}

Project numbers: SL13-FY13-187-PD03, OR13-CldBasedRIIDS-PD03

\section{INTRODUCTION}

A significant limitation in the operational utility of handheld and backpack radioisotope identifiers (RIIDs) is the ability of their onboard algorithms to accurately and reliably identify the isotopic sources of the measured gamma-ray energy spectrum and thereby separate threat from benign or background sources. Improved algorithms exist but are not implemented on RIIDs due to insufficient computational power or classification, export controls, or other sensitivities. They also have limited ability to use additional contextual information in making a threat no-threat assessment. A possible solution is to move the spectral analysis computations to an external device whose capabilities can be greatly enhanced and whose algorithms can be kept secure. A Cloud-based analysis system can provide this capability. Cloud computing is becoming ubiquitous and affords the national security community an opportunity for tremendous leverage of private sector developments. The goal of this project is to determine if operational performance of RIIDs can be improved by applying these developments in a way that meets the needs of the national security community.

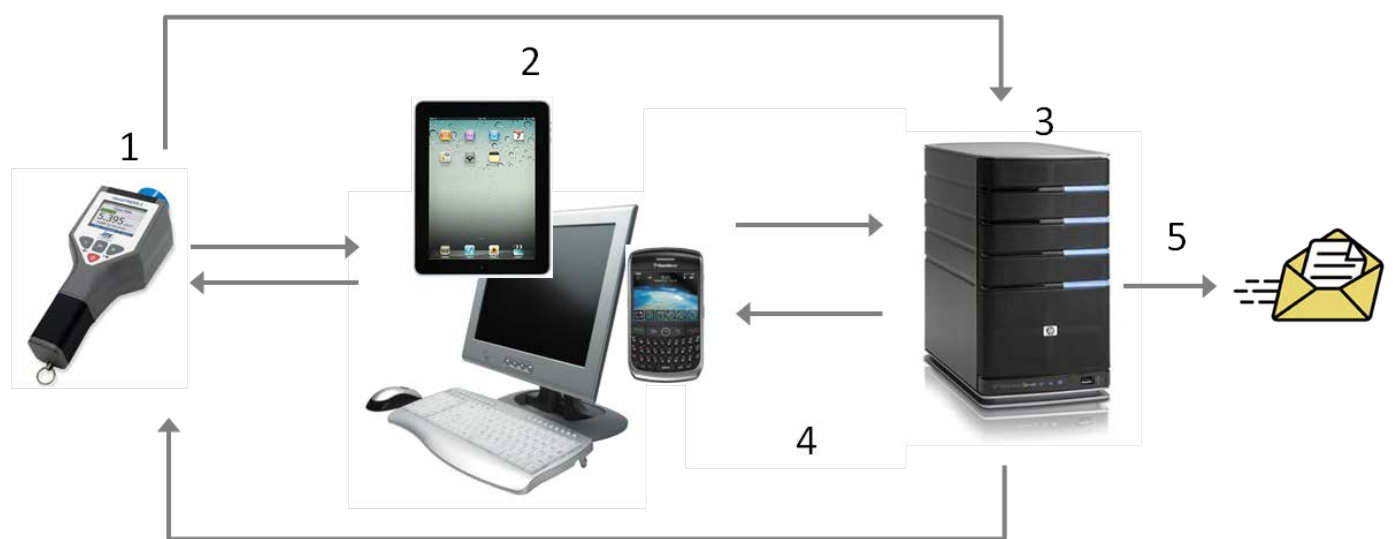

Figure 1. Example data flow of a Cloud-based RIID architecture. The portable detector (1) is linked to a bridge device (2) which collects the data and sends it to the Cloud server (3) which analyzes the data and sends the results back to the portable detector operator and to other interested parties (5).

The Cloud is intended to integrate with, not replace, existing information systems such as NA-42's Search Management Center (SMC) or DNDO’s Joint Analysis Center Collaborative Information System (JACCIS). Humans remain in the loop, and onboard analysis capability can remain on the devices as a backup.

\section{USER NEEDS FOR A CLOUD-BASED RIID SYSTEM}

A systems study was performed to look at the radiation detection mission architecture and determine the benefits, risks, and costs, as well a guide for development, of a Cloudbased RIID system. The Cloud system would optimize the already substantial amount of money that has been spent to train and equip federal, tribal, state, and local agencies in order to increase the system capabilities without placing additional burdens on the users. 
The impact of a Cloud-based RIID analysis capability was considered for the various users of such a system, as well as the necessary steps and objectives required for its implementation. Various interested parties were interviewed and observed in order to assess potential benefits and impacts on field operations of a cloud based analysis system. Two investigators on this project attended RAPTER to understand CONOPS and tool usage and to interface with RAP team members. First responders want quick adjudication and quick rejection of clearly innocuous alarms, compatibility with existing CONOPS, easier communications, retention of onboard capabilities supplemented by reanalysis by the Cloud, and field operation readiness. Operation managers want high-confidence adjudication, robust and accurate decision making tools, and the ability to train and guide first responders to take accurate data. Data analysts want streamlined data transfer for rapid adjudication, fusion of nonspectral data into the analysis, the ability to promote good data collection in the field, and training via the use of historical data and analysis.

\section{ADVANTAGES OF CLOUD-BASED ALGORITHMS}

Direct linking of RIIDs to the Cloud offers a number of advantages over the built-in capabilities currently available on the detectors, including the following.

- Improved Analysis - More accurate analysis of the measured data, using software and algorithms that exceed the capabilities of the built-in algorithms

- Streamlined Operation - Ability to directly interact with centralized information systems used by team leaders, incident commanders, headquarters, or analysts

- Easy updating - Ability to more easily update and maintain the analysis engine, without requiring firmware upgrades by the manufacturer

- Customizable analysis - Ability to customize the user interface or the algorithm parameters to the specifics of a user or situation, while still relying on the same advanced analysis engine on the back end

- Data fusion - Ability to incorporate nonspectral information into the analysis

- Monitoring of RIIDS - Ability to track device firmware, manage energy calibration and detector characterization, history and state of health, and provide on-line help and instructions

- Information Security - Algorithms can contain or use sensitive information that is not appropriate for wide dissemination on devices themselves, while the reported results may be sanitized of these sensitivities.

\section{DEMONSTRATION OF A CLOUD-BASED ANALYSIS}

A small-scale Cloud-based analysis system demonstrating most of the features of the architecture diagrammed in Figure 2 was implemented to show the feasibility and to gain practical experience. The system was demonstrated using a number of widely deployed COTS systems (FLIR identiFINDER, ORTEC Detective, Canberra Falcon 5000), as well as a custom-built detection system, to take data and send it to the cloud for analysis. The DHSIsotopeID and HPGeFSA algorithms were used to analyze the data with detector characterizations specialized for the individual detectors that submitted the data. Analysis results were then returned to the detector, in the case of the Falcon and the custom device, which had the ability to receive results onboard. For the other systems the results could be viewed on smartphones, tablets, or laptop computers. Using the web interface, 
additional information could be added to the analysis, the full spectrum regression fits could be viewed, and the data could be downloaded for further analysis.

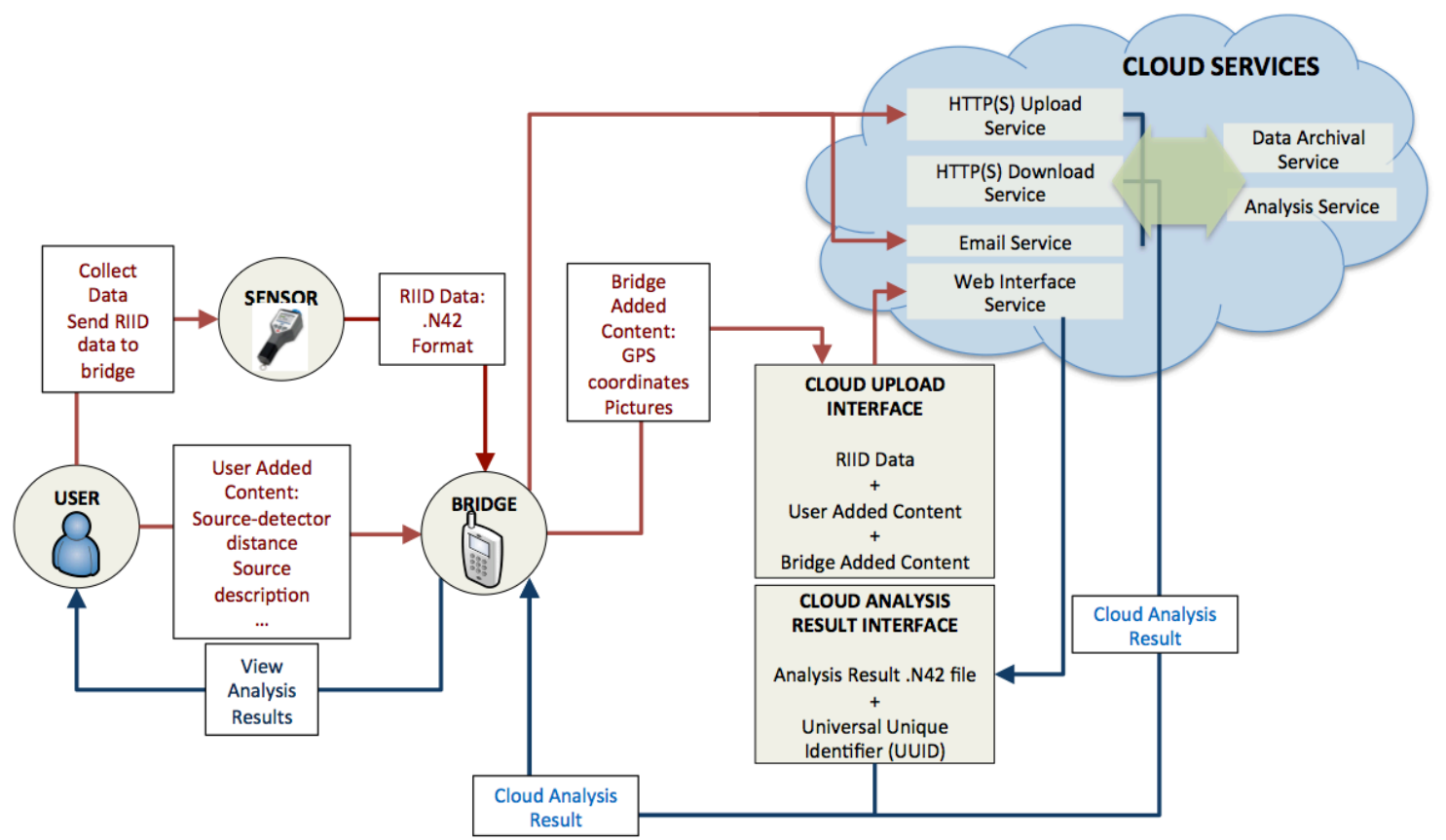

Fig. 2. Diagram of a potential Cloud-based RIID architecture.

\section{RESULTS, DISCUSSION, AND CONCLUSIONS}

The implementation and demonstration of a prototype Cloud-based RIID analysis system have shown this to be feasible with currently available communication and computational technology. The system study has shown that the potential user community could derive significant benefit from an appropriately implemented Cloud-base analysis system and has identified the design and operational characteristics required by the users and stakeholders for such a system.

\section{PATH FORWARD}

Integration of the Cloud architecture and analysis algorithms into existing systems such as the NA-42 Search Management Center (SMC) is the first step of wider implementation. This integration will be tested by actual field operations teams and must operate at the same or an increased speed of current CONOPS. More advanced, cloudspecific analysis algorithms will be integrated into the cloud system after rigorous verification and validation. A spiral development process will be used to prioritize implementation of new features based on results of the user testing and evaluation.

\section{PRESENTATIONS AND PUBLICATIONS}

An initial demonstration was given at the WMS 2013 poster session. A final description and demonstration was provided at RSL Andrews. The project final report was issued as a Sandia Report and an ORNL TM. 